\title{
Molecular and pathological basis of aceruloplasminemia
}

\author{
SATOSHI KONO and HIROAKI MIYAJIMA
}

First Department of Medicine, Hamamatsu University School of Medicine, 1-20-1 Handayama, Hamamatsu 431-3192, Japan

\begin{abstract}
Aceruloplasminemia is an autosomal recessive neurodegenerative disease characterized by iron accumulation in the brain as well as visceral organs. It is a loss-of-function disorder caused by mutations in the ceruloplasmin gene. Clinically, this disease consists of the triad of adult-onset neurological disease, retinal degeneration and diabetes mellitus. Massive iron accumulation and extensive loss of neurons are observed in the basal ganglia. The elevated iron concentration is associated with increased lipid peroxidation in the brains of aceruloplasminemia patients. Enlarged or deformed astrocytes and spheroid-like globular structures are characteristic neuropathological findings in aceruloplasminemia. Moreover, deformed astrocytes and globular structures react positively to anti-4-hydroxynonenal antibody, suggesting that increased oxidative stress is involved in neuronal cell death in aceruloplasminemia brain. More than 30 aceruloplasminemia-causing mutations in the ceruloplasmin gene have been identified. We examined the biosynthesis of two missense ceruloplasmin proteins that result from a Japanese P177R mutation and a Dutch G631R mutation, using Chinese hamster ovary cell expression system. The P177R mutant protein is retained in the endoplasmic reticulum. The G631R mutant protein, predicted to alter the interactions at a single type I copper-binding site, prevented incorporation of copper into apoceruloplasmin and resulted in the synthesis and secretion only of apoceruloplasmin. Molecular analysis of missense mutations showed different structure-function relationships in ceruloplasmin protein. The investigation of mutant ceruloplasmin reveals new insights into molecular pathogenesis of aceruloplasminemia as well as biosynthesis, trafficking, and function of ceruloplasmin.
\end{abstract}

Key terms: ceruloplasmin, iron; lipid peroxidation, mutation

Ceruloplasmin (CP) is an alpha-2glycoprotein that contains more than $95 \%$ of the copper in the plasma. This protein is a multicopper oxidase with six atoms of copper incorporated and plays a role in the mobilization and oxidation of iron from tissue stores with subsequent incorporation of ferric iron into transferrin (Osaki et al 1966). It also has antioxidant effects through the oxidation of ferrous iron. CP is mainly synthesized in hepatocytes and also is expressed in the central nervous system. In the brain, most of the CP is located on the surface of astrocytes in a glycosylphosphatidylinositol (GPI)anchored form (Patel \& David, 1997; Patel et al., 2000). This protein plays an essential role for brain iron metabolism (Jeong \& David, 2003). The importance of CP in human biology is underscored by the autosomal recessive disorder aceruloplasminemia that is an inherited disease of iron homeostasis. In 1987, Miyajima first described a 52-year-old Japanese woman suffering from blepharospasm, retinal degeneration, and diabetes mellitus (Miyajima et al., 1987). Her symptoms were associated with a complete absence of circulating serum $\mathrm{CP}$. DNA sequence analysis of this patient showed 5 base insertion in exon 7 within the $\mathrm{CP}$ gene, resulting in a frame shift mutation and a truncated open reading frame (Harris et al., 1995). Clinical findings and identification of a mutation in the $\mathrm{CP}$ gene suggested a novel disorder of iron metabolism due to a lack of CP. We termed this disease 'aceruloplasminemia,' More than 40 families affected from aceruloplasminemia have now been 
reported. Clinical manifestations of aceruloplasminemia are included in the triad of retinal degeneration, diabetes mellitus and neurological symptoms (Miyajima et al., 2003). Neurological symptoms include cerebellar ataxia, involuntary movement, parkinsonism, and cognitive dysfunction. Characteristic pathological features of aceruloplasminemia are progressive neurodegeneration of basal ganglia in association with iron accumulation in these tissues. CP may play an important role in normal brain iron homeostasis. Under normal circumstances, circulating $\mathrm{CP}$ dose not cross the blood-brain barrier. Iron is taken into the brain tissues from the circulation through receptor-mediated endocytosis of transferrin. Iron may be oxidized by GPI-linked CP expressed in the astrocytes, incorporated into transferrin derived from the oligodendrocytes, and transferred to the neuronal cells. In the absence of $\mathrm{CP}$, iron is accumulated in the brain cells. In this paper, we describe neuropathological features and molecular pathogenesis of aceruloplasminemia.

\section{Neuropathology of aceruloplasminemia}

We examined five brains from patients with aceruloplasminemia histopathologically and immunohistochemically (Kaneko et al., 2002). Macroscopic findings of the patient's brains show a mild degree of cortical atrophy. The cerebellum, tegmentum of the midbrain, and pons appeared mildly shrunken. Coronal sections of the basal ganglia, thalamus, and dentate nucleus of the cerebellum demonstrated brownish pigmentation. Microscopic finding showed severe iron deposition in the astrocytes and nerve cells in the basal ganglia, thalamus and cerebellum. Neuronal loss was mild in the cerebellar cortex and substantia nigra with moderate iron accumulation (Table 1). The distribution of iron deposition was essentially the same pattern in all patients. In addition to iron deposition and neuronal cell loss, the most characteristic histopathological findings observed in patients with aceruloplasminemia were abnormal deformed astrocytes and globular structures. Both findings were seen more frequently in the striatum than in the frontal cortex (Table 1). In the basal ganglia, many astrocytes were enlarged in size with abundant cytoplasm and prominent nuclei. Some giant astrocytes with large, multiple or lobulated nuclei were observed in the caudate nucleus and putamen (Fig. 1A). An immunohistological study using antibody against glial fibrially protein (GFAP), an astrocyte specific protein, showed strong signals within the cytoplasm and on the processes of the cells (Fig. 1C). These astrocytes were also positive to anti-4hydroxynoneal (HNE) antibody that is a marker of lipid peroxidation (Fig. 1D). A number of globular structures also were identified in the superficial layers of frontal cortex and in the basal ganglia. These structures were seen in proportion to the degree of iron deposition. The globular structures varied in size from 10 to 60 micrometers in diameter. Many structures showed the grumose internal structure and contained faintly eosinophilic materials (Fig. 2A) and iron deposition (Fig. 2B).

TABLE I

Histopathological Summary of Five Aceruloplasminemia Patients

\begin{tabular}{lcccc}
\hline & Frontal cortex & Caudate nucleu & Putamen & Globus pallidus \\
\hline Iron deposition & + & +++ & +++ & + \\
Nerve cell loss & +++ & +++ & +++ & + \\
Abnormal astrocytes & ++- & + & + & $+\sim-$ \\
Glubular estructure & $+\sim-$ & +++ & ++ & ++ \\
& & & & + \\
\hline
\end{tabular}




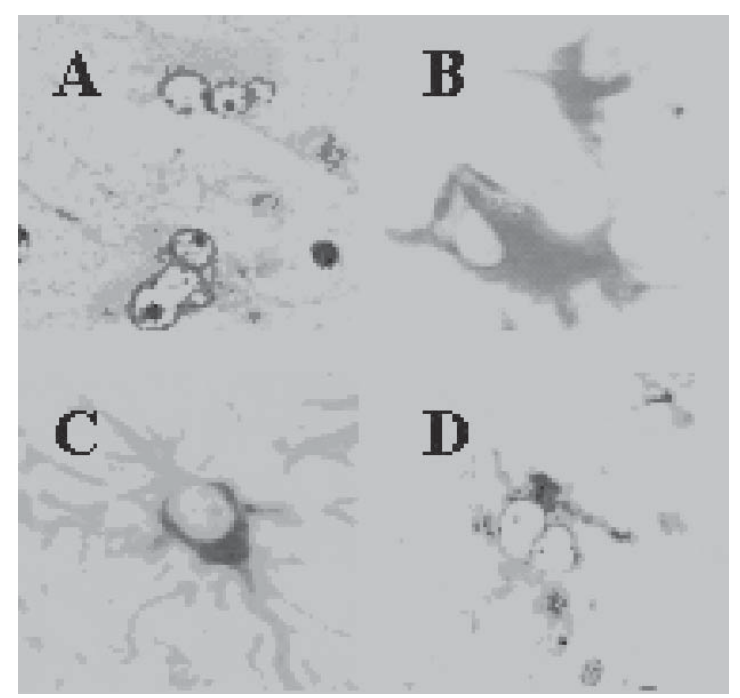

Figure 1. Deformity of astrocytes in aceruloplasminemia brains. Giant astrocytes with large lobulated nuclei or a few nuclei were seen in the caudate nucleus and putamen (A). Cytoplasm of the abnormal astrocytes shows marked iron deposition (B) and reacts to antiGFP antibody(C) and anti-HNE antibody (D).
Immunohistochemically, many globular structures were positive to anti-GFAP antibody (Fig. 2C) and anti-S-100 antibody (Fig. 2D), but not to anti-neurofilament (Fig. 2E) or anti-synaptophysin antibody (Fig. 2F). These results suggested that the structures were composed of substances mainly derived from astrocytes. Therefore, the globular structures differed from common spheroids, which were thought to be axonal swellings of neurons. In addition, these structures reacted with anti-HNE (Fig. 2H) and anti-ubiquitin antibodies (Fig. 2G). These findings suggest that oxidative stress is increased in the brains of aceruloplasminemia patients, and these structures include many oxidatively damaged and ubiquitin-conjugated proteins. The reason why the abnormal astrocyte appears in the brains of aceruloplasminemia patients is unclear. This finding may be related to iron-induced tissue damage, since these abnormal astrocytes were observed more frequently in the basal ganglia where marked iron accumulation was seen.

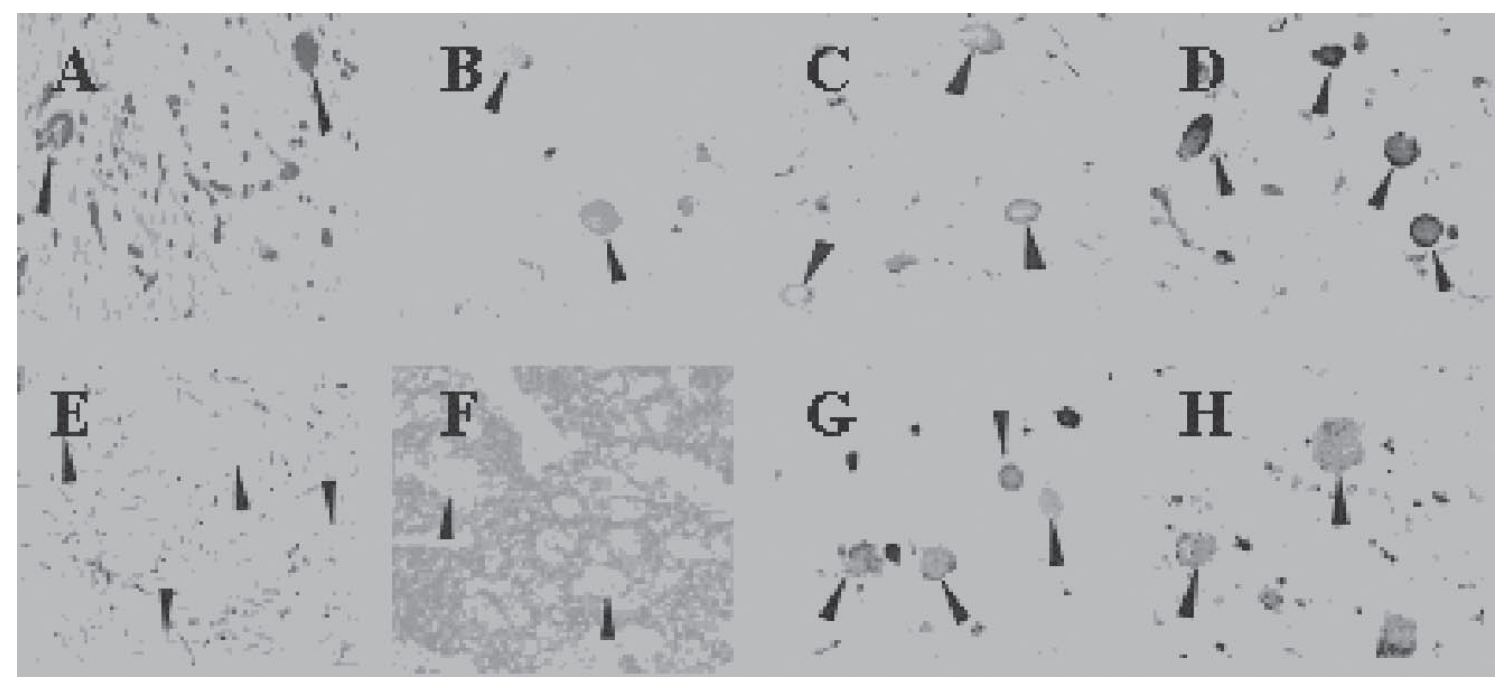

Figure 2. Globular structure in aceruloplasminemia brains. The globular structures have a grumose appearance and contain eosinophilic material (A) and iron deposition (B). This structure reacts to anti-GFP antibody(C), anti-S100 antibody(D), anti-ubiquitin antibody (G), anti-HNE antibody (H), but not to anti-neurofilament antibody(E) or anti-synaptophysin antibody (F). 
Antioxidant activity of $\mathrm{CP}$ can be ascribed mainly to its ferroxidase activity, which inhibits ferrous ion-stimulated lipid peroxidation and formation of hydroxyl radicals in the Fenton reaction. $\mathrm{CP}$ is not only a ferroxidase but also a scavenger of reactive oxygen species. In aceruloplasminemia, many reports showed a marked increase in lipid peroxidation in cerebral spinal fluid and brain tissues in patients (Kohno et al., 2000; Miyajima et al., 1998; Miyajima et al., 2001; Tajima et al., 1999; Yoshida et al., 2000). Iron accumulation and deformities in astrocytes are characteristic neuropathological features of this disease. The morphologic deformities in astrocytes are linked to oxidative stress induced by iron accumulation, and astrocyte dysfunction contributes to neuronal cell death in addition to the direct effects of free radicals on neurons (Scheme 1). Lipid peroxidation in astrocytes caused both by free radicals and excessive iron may participate in the pathogenesis of this disease.

\section{Biosynthesis of mutant ceruloplasmin}

More than thirty aceruloplasminemiacausing mutations have been identified (Scheme 2). Some of the mutations have been found in several families with aceruloplasminemia, while others have been detected in a single patient. The majority of mutations in the $\mathrm{CP}$ gene are the truncated mutations leading to the formation of a premature stop codon. These mutations would be predicted to result in formation of a protein lacking the copper-binding sites presumed to be critical for enzymatic function. To investigate the molecular pathogenesis of aceruloplasminemia, the biosynthesis of missense mutant $\mathrm{CP}$ was examined.

Copper enters the cell via copper transport proteins, Ctrl and Ctr2, and is then bound either to the metal storage protein, methalthionein, or to one of the copper chaperons, Cox17, CCS, and Atox1, which deliver copper to its appropriated targets. Atox-1 delivers copper to the secretory pathway, and then copper is pumped into the lumen of the endoplasmic reticulum by the Wilson/Menkes protein. Copper containing proteins in the secretory pathway such as $\mathrm{CP}$ are initially synthesized as an apo-protein and obtain copper to change from an apoform (non copper binding form) to a copper binding holo-form (all six copper atoms binding form). The secreted $\mathrm{CP}$ consisted of 1046 amino acids is synthesized in mainly hepatocytes as both apo- and holo-forms. The apo- and holo-forms of CP migrate differentially (Fig. 3A). When the $\mathrm{CP}$ is subjected to SDS-PAEG electrophoresis, apo-CP runs as $135 \mathrm{kDa}$ band and holo- $\mathrm{CP}$ as an $85-\mathrm{kDa}$ band (Sato \& Gitlin, 1991).

SCHEME 1

Putative Pathogenesis of Aceruloplasminemia Ceruloplasmin deficiency

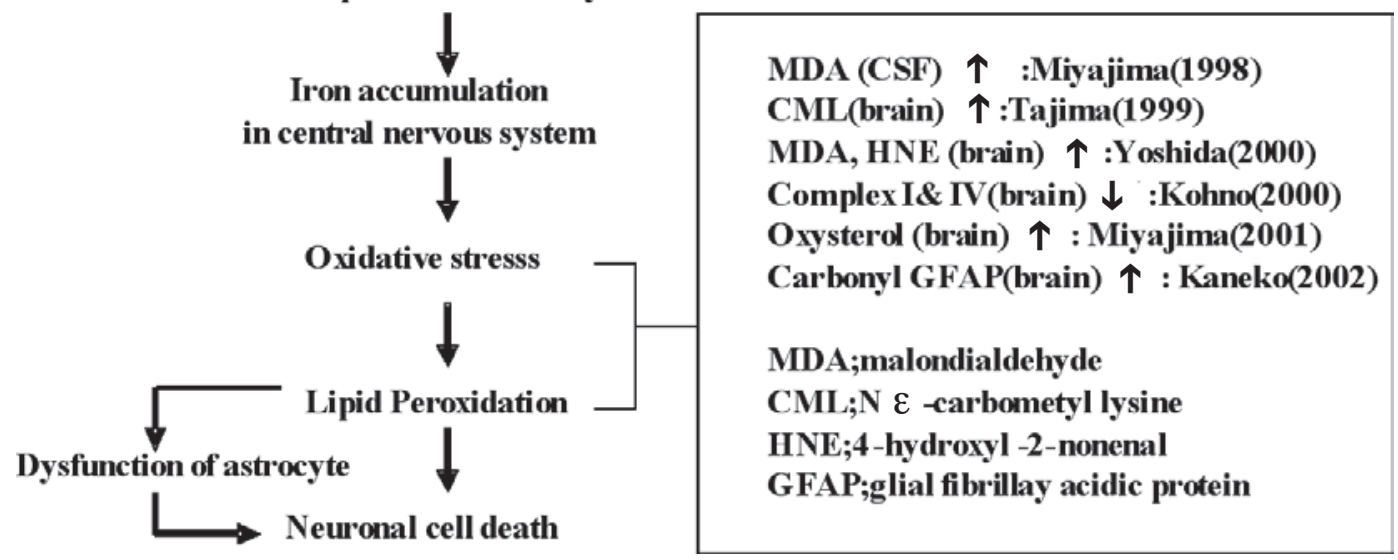


Aceruloplasminemia-causing mutations in ceruloplasmin gene

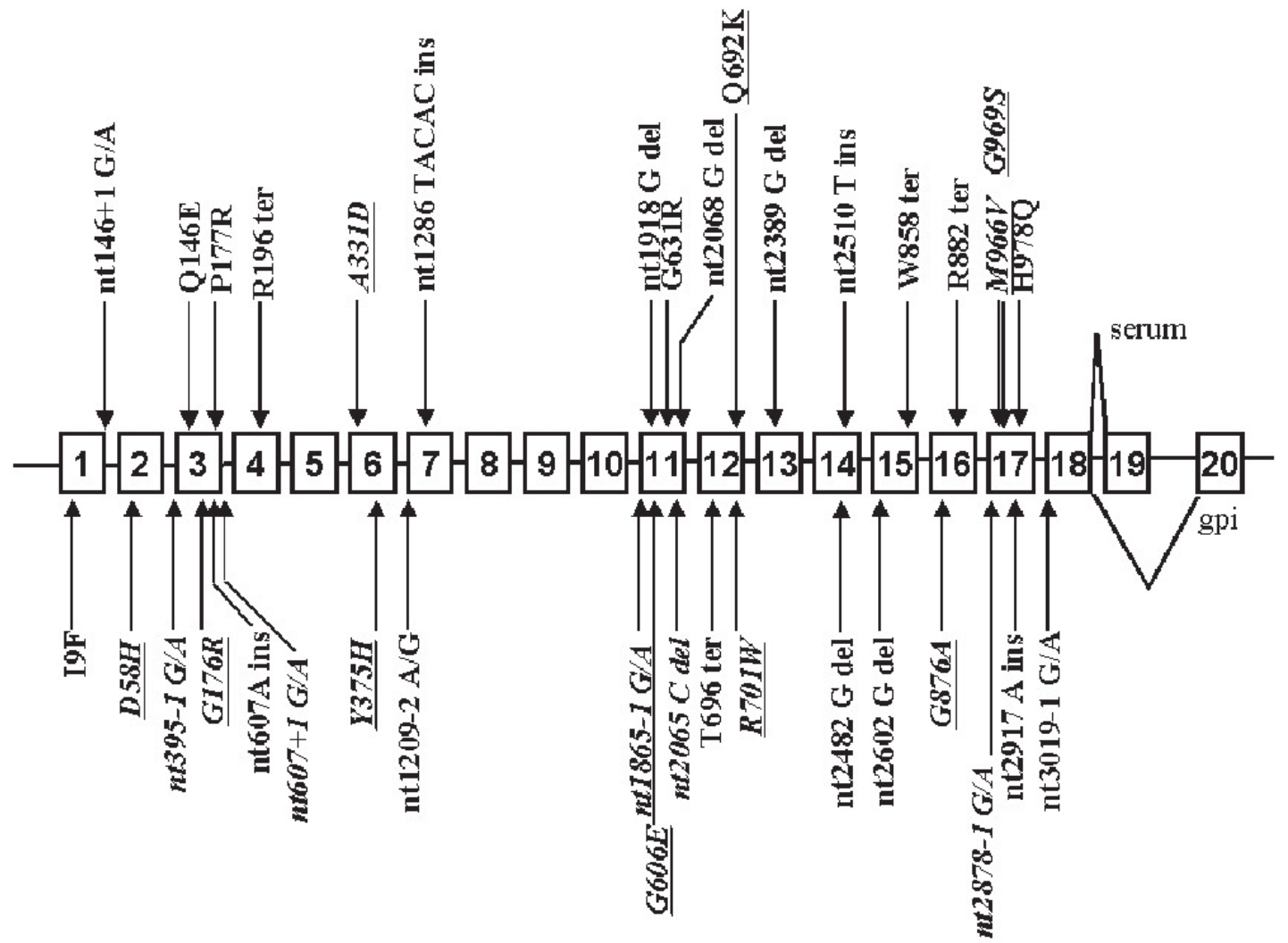

We transfected cDNA encoding human $\mathrm{CP}$ into Chinese hamster ovary cell line ( $\mathrm{CHO}$ cells), because no endogenous $\mathrm{CP}$ synthesis was detected in these cells. A missense P177R mutant was examined for the effect of copper incorporation into $\mathrm{CP}$ (Hellman et al., 2002b). Transfected CHO cells were analyzed by immunoblot using anti-CP antibody and metabolically labeled $\mathrm{Cu}-64$ for detection of copper-labeled CP in the media. An $85 \mathrm{kDa}$ band corresponding to holo-CP and a $135 \mathrm{kDa}$ band corresponding to apo-CP were detected in the media of wild-type transfected $\mathrm{CHO}$ cells, but both bands were not detected in the media of the missense mutation (Fig. 3B). We examined gene expression of mutant $\mathrm{CP}$ cDNA transfected into $\mathrm{CHO}$ cells by $\mathrm{S} 35$ pulse-chase analysis. $\mathrm{CHO}$ cells transfected with wild-type or mutant $\mathrm{CP}$ were incubated with S35 labeled methionine and cysteine and chased in media for the indicated periods of time. CP was immunoprecipitated from cell lysates and media and analyzed using SDS-PAGE. Newly synthesized protein of mutant CP showed intracellular accumulation without degradation and was not secreted to the media throughout the time course of the experiment (Fig. 3C). Immunofluorescence staining was performed in $\mathrm{CHO}$ cells transfected with the wild-type and P177R GPI-linked constructs. GPI-linked CP displays a staining pattern consistent with cell surface localization. In contrast, the GPI-linked form of the missense CP is detected in an intracellular compartment of the endoplasmic reticulum (ER) colocalized with resident protein disulfide isomerase (PDI) (Fig. 3D). This data suggested that the P177R mutant protein may be trafficked improperly in the ER. 

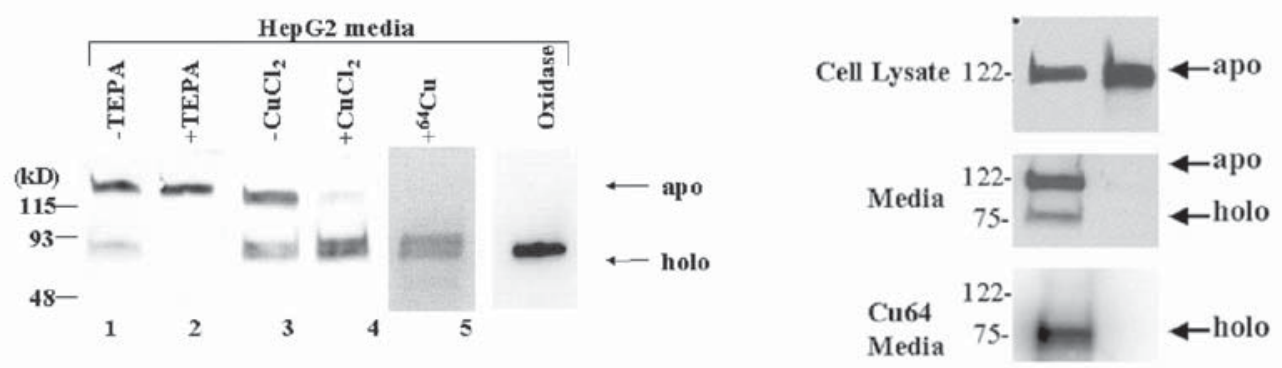

D
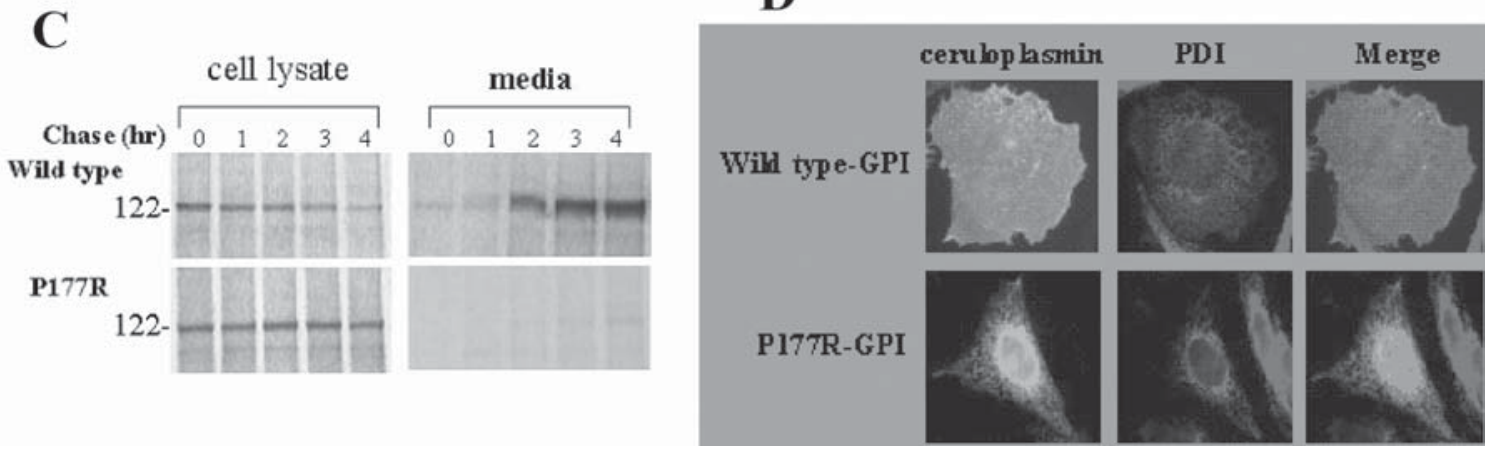

Figure 3. A. Apo- and holo-CP in media secreted from HepG2 cell line. HepG2 cells were incubated with serum-free media with nothing (lane 1, 3), 100 $\mu \mathrm{M}$ TEPA (Cu chelatortetrahylenepentamine (TEPA) (lane 2), $100 \mu \mathrm{M} \mathrm{CuCl}_{2}$ (lane 3), or $100 \mu \mathrm{Ci} / \mathrm{ml} \mathrm{Cu}-64$ (lane 5). Bands were visualized either by immunodetection with anti-human $\mathrm{CP}$ antibody (lane $1-4),{ }^{64} \mathrm{Cu}$ labeling (lane 5), or oxidase stain (lane 6). B. Chinese hamster ovary (CHO) cells transfected with either wild-type or P177R CP was incubated with $100 \mu \mathrm{Ci} / \mathrm{ml} \mathrm{Cu}-64$. Media and cell lysates sample were run on $4-15 \%$ Tris- $\mathrm{HCl}$ gradient gels and analyzed by immunodetection of $\mathrm{CP}$ using anti-human $\mathrm{CP}$ antibody. C. CHO cells transfected with wild-type or P177R CP cDNA were incubated with S35labeled methionine and cystein for $20 \mathrm{~min}$ and chased in the media for the indicated period. Recombinant CP was immunoprecipitated from cell lysates and media and run on $7.5 \%$ SDS-PAEG gel. D. Indirect immunofluorescence localization of CP in CHO cells transfected GPI-linked CP or GPI-linked P177R CP using antibody against CP (green) and protein disulfide isomerase (red).

In the amino acid sequence of human $\mathrm{CP}$, we found that the P177R mutation resides within a five amino acid motif $\mathrm{G}(\mathrm{FLI})(\mathrm{LI}) \mathrm{GP}$ that is repeated six times throughout the protein. This motif is also present and repeated in the homologous multicopper oxidase hephaestin as well as coagulation factor VIII. An analogous proline to arginine mutation in this motif has been identified in the factor VIII gene in a patient with hemophilia A (Antonarakis et al., 1995). The repeated motif may be critical for proper folding and subsequent trafficking of human $\mathrm{CP}$.
We recently identified a new missense mutation in the $\mathrm{CP}$ gene in a Dutch family with aceruloplasminemia (Hellman et al., 2002a). The proband in this family had no serum CP and was diagnosed as spinocerebellar degeneration. Genetic analysis of the CP gene showed that it was homozygous for the mutation G631R. When the G631R mutation was subjected to S35 pulse-chase analysis, CP protein was synthesized and secreted, and its protein was identical to wild-type $\mathrm{CP}$ by amino acid sequence analysis. By the immunobloting analysis, however, this 
mutation resulted in a failure to bind $\mathrm{Cu} 64$, and the G631R mutant protein existed as the apo-form (Figure 4A). Electrophoresis might disladge copper bound to the mutant incorporating the copper might be less stable than wild-type ceruloplamin. To examine this hypothesis, we used sizeexclusion chromatography to determine the Cu64 incorporation into the mutants. Media from Cu64-labeled CHO cells transfected with either wild-type or mutant $\mathrm{CP}$ was passed over a size-exclusion column. Collected fractions were assayed for $64 \mathrm{Cu}$ radioactivity in a gamma counter as well as for $\mathrm{CP}$ content. The media from Cu64labeled $\mathrm{CHO}$ cells transfected with wildtype CP showed a peak in the Cu64 elution profile in fractions 7-9. However, $\mathrm{CHO}$ cells transfected with G631R mutant CP did not show such a peak in their respective Cu64 elution in fractions 7-9, but abundant apo-CP was eluted from these fractions (Figure 4B). These data suggested that copper incorporation into $\mathrm{CP}$ was prevented in the G631R mutant protein. This mutation would influence copper-binding site either

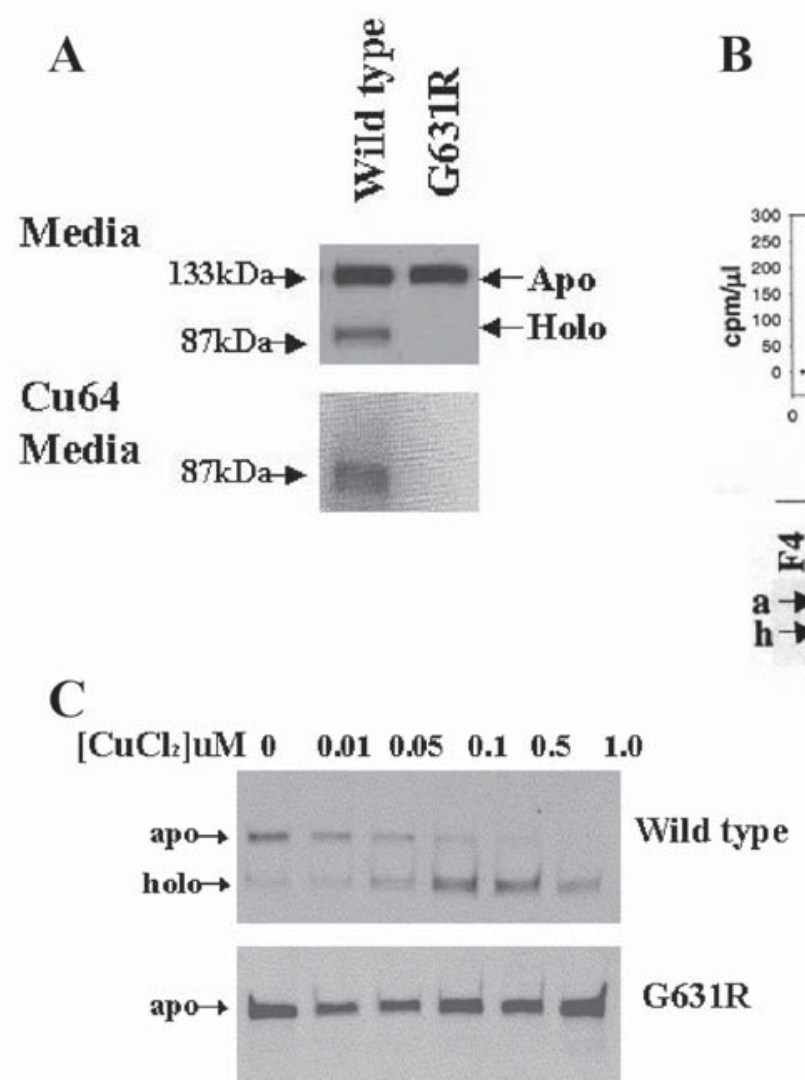

Figure 4. A. Immunoblot analysis and $\mathrm{Cu}-64$ incorporation study of media from $\mathrm{CHO}$ cells transfected with either wild-type or G631R CP. B. Cu-64 elution study of media collected from CHO cells transfected with either wild-type or G631R CP. Media from Cu64-labled cells was passed over a Sephacryl S-100 size exclusion column. Radioactive counts from each fraction were expressed as counts per minute $(\mathrm{cpm} / \mu \mathrm{l})$ with a gamma counter. Immunoblot analysis of fraction 412 media samples using anti-CP antibody. The presence of apo- and holo-CP is indicated with arrows as "a" and "h". C. In vitro copper incorporation into CP. CHO cells transfected with either wild-type or G631R mutant CP were incubated with $100 \mu \mathrm{M}$ TEPA. Media from these cells was added $\mathrm{pH} 5.7$ sodium acetate buffer containing varying amounts of $\mathrm{CuCl}_{2}$ and analyzed by immunobloting with anti-CP antibody. 
SCHEME 3

Molecular mechanism of aceruloplasminemia

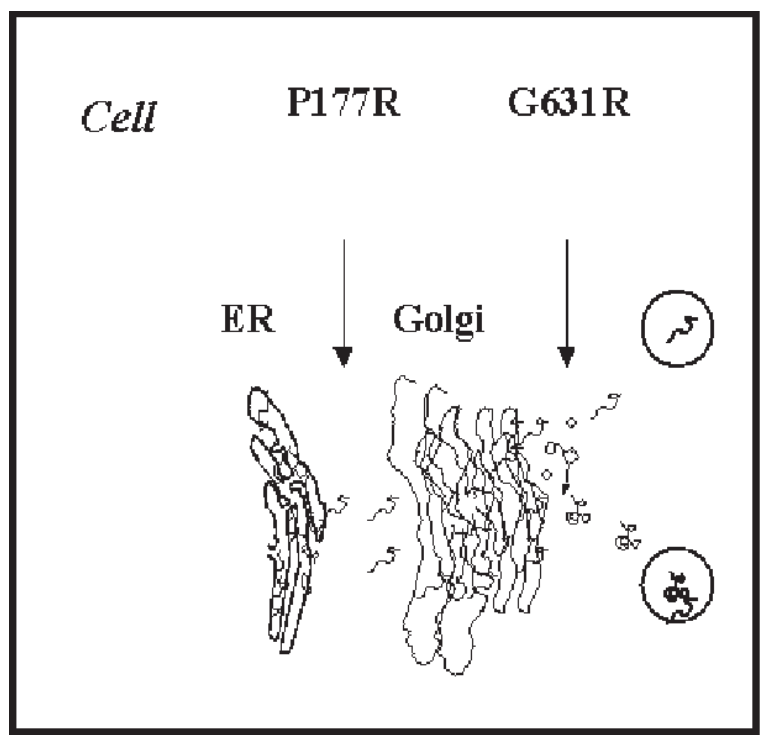

s apoceruloplasmin

$\because \mathrm{Cu}$
Extra-cellular space

wild type

(f) $\rightarrow$ apo-ceruloplasmin

(d) holo-ceruloplasmin directly or indirectly. Media from either wild-type or mutant $\mathrm{CP}$ was added to copper exchange buffer containing varying amounts of $\mathrm{CuCl}_{2}$. As the amount of $\mathrm{CuCl}_{2}$ was increased, wild-type apo-CP gradually increased the ratio of holo to apo until all of the apo-CP had been converted into holo$\mathrm{CP}$. G631R protein remained in the apoform even at the highest level of $\mathrm{CuCl}_{2}$ (Fig. 4C). This data demonstrates that the inability of mutant $\mathrm{CP}$ to obtain copper is a property of the protein itself, rather than a defect in intracellular trafficking or chaperone binding in vivo.

Molecular analysis of these missense mutations has shown several different mechanisms by which mutations in the CP gene can result in the lack of enzymatic activity (Scheme 3). As P177R mutant protein is retained in the endoplasmic reticulum, aceruloplasminemia is one of diseases caused by defects in protein trafficking. On G631R mutation analysis,
CP deficiency can arise through other mechanisms, either by indirect dysfunction of a copper-binding site or by other structural abnormalities in the protein that prevent the incorporation of copper into $\mathrm{CP}$.

\section{ACKNOWLEDGEMENTS}

We thank our coworkers, Drs Y Takahashi, First Department of Medicine, Hamamatsu University School of Medicine; K Kaneko, K Yoshida, S Ikeda, Third Department of Internal Medicine, Shinshu University School of Medicine; M Daimon, T Kato, Third Department of Internal Medicine, Yamagata University, School of Medicine; GM Mancini, AJ Hoogeboom, GJ de Jong, Department of Clinical Genetics, Erasmus University; and JD Gitlin, NE Hellman, Department of Pediatrics, Washington University School of Medicine in St. Louis, for their collaboration. 


\section{REFERENCES}

ANTONARAKIS SE, KAZAZIAN HH, TUDDENHAM EG (1995) Molecular etiology of factor VIII deficiency in hemophilia A. Hum Mutat 5: 1-22

HARRIS ZL, TAKAHASHI Y, MIYAJIMA H, SERIZAWA M, MACGILLIVRAY RT, GITLIN JD (1995) Aceruloplasminemia: Molecular characterization of this disorder of iron metabolism. Proc Natl Acad Sci USA 92: 2539-43

HELLMAN NE, KONO S, MANCINI GM, HOOGEBOOM AJ, DE JONG GJ, GITLIN JD (2002a) Mechanisms of copper incorporation into human ceruloplasmin. J Biol Chem 277: 46632-8

HELLMAN NE, KONO S, MIYAJIMA H, GITLIN JD (2002b) Biochemical analysis of a missense mutation in aceruloplasminemia. J Biol Chem 277: 1375-80

JEONG SY, DAVID S (2003) Glycosylphosphatidylinositolanchored ceruloplasmin is required for iron efflux from cells in the central nervous system. J Biol Chem 278: 27144-8

KANEKO K, YOSHIDA K, ARIMA K, OHARA S, MIYAJIMA H, KATO T, OHTA M, IKEDA S (2002) Astrocytic deformity and globular structures are characteristic of the brains of patients with aceruloplasminemia. J Neuropathol Exp Neurol 61: 1069-77

KOHNO S, MIYAJIMA H, TAKAHASHI Y, SUZUKI H, HISHIDA A (2000) Defective electron transfer in complexes I and IV in patients with aceruloplasminemia. J Neurol Sci 182: 57-60

MIYAJIMA H, ADACHI J, KOHNO S, TAKAHASHI Y, UENO Y, NAITO T (2001) Increased oxysterols associated with iron accumulation in the brains and visceral organs of acaeruloplasminaemia patients. Qjm 94: 417-22
MIYAJIMA H, FUJIMOTO M, KOHNO S, KANEKO E, GITLIN JD (1998) CSF abnormalities in patients with aceruloplasminemia. Neurology 51: 1188-90

MIYAJIMA H, NISHIMURA Y, MIZOGUCHI K, SAKAMOTO M, SHIMIZU T, HONDA N (1987) Familial apoceruloplasmin deficiency associated with blepharospasm and retinal degeneration. Neurology 37 : 761-7

MIYAJIMA H, TAKAHASHI Y, KONO S (2003) Aceruloplasminemia, an inherited disorder of iron metabolism. Biometals 16: 205-13

OSAKI S, JOHNSON DA, FRIEDEN E (1966) The possible significance of the ferrous oxidase activity of ceruloplasmin in normal human serum. J Biol Chem 241: $2746-51$

PATEL BN, DAVID S (1997) A novel glycosylphosphatidylinositol-anchored form of ceruloplasmin is expressed by mammalian astrocytes. J Biol Chem 272: 20185-90

PATEL BN, DUNN RJ, DAVID S (2000) Alternative RNA splicing generates a glycosylphosphatidylinositolanchored form of ceruloplasmin in mammalian brain. $\mathrm{J}$ Biol Chem 275: 4305-10

SATO M, GITLIN JD (1991) Mechanisms of copper incorporation during the biosynthesis of human ceruloplasmin. J Biol Chem 266: 5128-34

TAJIMA K, KAWANAMI T, NAGAI R, HORIUCHI S, KATO T (1999) Hereditary ceruloplasmin deficiency increases advanced glycation end products in the brain. Neurology 53: 619-22

YOSHIDA K, KANEKO K, MIYAJIMA H, TOKUDA T, NAKAMURA A, KATO M, IKEDA S (2000) Increased lipid peroxidation in the brains of aceruloplasminemia patients. J Neurol Sci 175: 91-5 